\title{
Improving Teaching Quality Based on App Virtual Simulation Technology
}

\author{
Pengfei Cao ${ }^{1, a^{*}}$, Guanmao Zhang ${ }^{1, b}$, Lin Cheng ${ }^{1, \mathrm{c}}$ \\ ${ }^{1}$ School of Information Science and Engineering, Lanzhou University, Lanzhou, 730000, China \\ a*caopf@lzu.edu.cn \\ ${ }^{b}$ zhanggm@lzu.edu.cn \\ cchenglin@lzu.edu.cn
}

\begin{abstract}
With the COVID-19 pandemic, the large-scale online course construction of "no suspension of classes" is presented by the Ministry of education of the People's Republic of China. For the teaching of science and engineering courses, the current online teaching lacks corresponding teacher-student interaction, especially the lack of experimental supporting and directly portraying various physical phenomena, which is adverse to deepening students' understanding and stimulating learning and innovation capabilities. Virtual simulation is conducive to the open sharing of teaching resources, which can solve many problems such as the disconnection between online theoretical teaching and experimental classroom, and the shortage of experimental instruments. According to the new online teaching methods, taking into account the challenges of virtual simulation experimental teaching platform construction and the difficulties of maintenance during the epidemic, combined with the characteristics of the "Principles of Optics" course and APP simulation applications, a set of effective teaching and experiment methods based on online virtual simulation technology are explored. It not only improves the teaching quality, but also promotes students' ability of active learning, which provides new ideas for improving the quality of traditional classroom teaching.
\end{abstract}

Keywords: Online teaching, virtual simulation, App application, Online experiment, simulation software

\section{App 虚拟仿真技术提高教学质量的探索}

\author{
曹鹏飞 ${ }^{1,} a^{*}$ 张冠茂 $^{1, b}$ 程琳 $^{1,}$ 。
}

${ }^{1}$ 兰州大学信息科学与工程学院, 甘肃, 兰州, 中国

a*caopf@lzu.edu.cn

bzhanggm@lzu.edu.cn

c chenglin@lzu.edu.cn

\section{摘要}

在新冠疫情下, 我国首先开展 “停课不停学” 的大规模在线课程建设。对于理工科课程教学, 目前的线上教学 缺乏相应的师生互动, 特别是缺乏实验的配套支撑, 不能直观刻画各种物理现象, 不利于加深学生的理解力以 及激发学习能力和创新能力。虚拟仿真有利于教学资源开放共享, 解决线上理论教学和实验课堂的脱节以及线 下多人一组实验仪器等教学资源不足的问题。根据新的线上教学与学习方式, 考虑到虚拟仿真实验教学平台建 设的挑战与疫情期间维护的困难, 结合 《光学原理》课程特点与 APP 仿真应用程序, 摸索出了一套行之有效的 基于线上虚拟仿真技术的教学和实验方法, 提高了教师授课质量的同时也推动了学生主动学习的能力, 这也为 提高传统课堂教学质量提供新思路。

关键词: 线上教学, 虚拟仿真, $A p p$ 应用程序, 线上实验, 仿真软件 


\section{1. 前言}

2020 年席卷全球的新冠肺炎使得人们的生产生 活产生了重大变化。全国各个高校积极响应教育部提 出的 “停课不停学” 开展在线授课的居家式学习, 教 学模式发生了重大变化 $[1,2]$ 。但是针对理工科教学 特点, 仅是线上讲授课程远远无法满足国家大力推动 的 “新工科” 建设的需要。以《光学原理》为例，传 统在线课堂讲授缺少了相应的实验课程配套支撑, 存 在如不能直观刻画光学所描述的各种现象, 不利于学 生加深对知识点的理解以及不能很好激学生的学习 能力和创新能力等缺陷, 将严重影响学生的培养质量。 因此, 如何充分提高线上教学效率, 激发学习热情, 提高师生之间的互动, 是当前线上课程建设和改革的 主要目标。

虚拟仿真是一种将传统的实验教学与互联网、计 算机等技术有机地融合在一起来实现各种虚拟实验 环境的技术 [3]。虚拟仿真有利于教学资源开放共享, 解决理论教学和实验课堂的脱节以及多人一组实验 仪器等教学资源不足的问题, 成为当今教育信息化改 革的发展潮流 [4], 也是目前解决线上教学不足的有 效手段。

然而, 考虑到虚拟仿真实验教学平台建设的挑战 [5] 以及在疫情期间维护的困难, 本文根据《光学原 理》课程特点, 提出基于 COMSOL Multiphyics 以及 App 封装的虚拟仿真平台。基于有限元法的 COMSOL Multiphyics 是一款多物理场的数值仿真软件 [6], 它实际上是从 Matlab 的偏微分工具箱发展而来, 目 前可在一个模型下建立多个物理场的耦合计算。鉴于 COMSOL Multiphyics 软件强大的仿真能力可将流体、 电磁场等抽象的物理问题具体化、可视化, 非常有利 于理工科学生理解、消化抽象知识点, 因而该软件作 为虚拟仿真平台逐步被引入到课堂教学中 [7]。通过 探索使用 COMSOL Multiphyics 软件 App 应用结合《光 学原理》课程内容，建立具体光学问题的线上虚拟仿 真平台。COMSOL Multiphyics 软件的 App 应用不仅 能够在 Windows Linux 和 Mac OS 操作系统上运行, 而且可以不用安装相应软件, 有效使没有仿真专业知 识的学生通过友好的用户界面设置测试参数进行计 算。这一创新性的技术手段可以弥补线上教学中缺乏 实验支撑的不足, 加深学生对课程中抽象知识的理解, 进一步提升线上教学的学习效果。

\section{2. 以环型谐振腔耦合原理为例的线上 App 虚 拟仿真教学}

在讲述研究生课程《光学原理》中的平面光波导 技术时 [8]，介绍了基于平面波导技术的光微环谐振 腔。因其独特的耦合特性受到人们的关注和研究而得 以迅速发展并成为构建和实现集成光子学功能器件 的重要的基础光波导单元 $[9,10]$ 。目前以光微环谐振 腔耦合机制实现的调制解调器可达到 $10 \mathrm{~Gb} / \mathrm{s}$ 的高速
调制。为了让学生对光微环谐振腔的耦合特性有直观 了解, 以经典的环型谐振腔耦合原理为例开展通过 COMSOL Multiphyics 软件的 App 应用的线上虚拟仿 真教学, 更加形象的让学生理解耦合原理。经典的环 型谐振腔耦合建模参见 COMSOL Multiphyics 案例库 波动光学模块下波导与耦合器中的《Optical Ring Resonator Notch Filter》。

\section{1. 环型谐振腔耦合原理}

光微环谐振器的基本结构包括一个封闭的回路 环形波导和回路旁边的一根或者两根总线波导，其中 回路可以是任何形状的圆、椭圆、跑道式; 总线波导 通常是直波导，作为输入输出信道。这两个波导的芯 层彼此靠近放置，如图 1 所示。其耦合原理是当输入 端输入一束光，在直波导内存在的光场一定满足高斯 解，也就意味着大部分能量被约束在波导内; 部分能 量在波导外以指数形式迅速衰减，这部分波被称为修 逝波。在两个波导相互靠近的区域，满足谐振条件时， 直波导的修逝波就会被耦合在环形波导内。当达到光 场平衡时，满足谐振条件的光信号在环形波导中累积 了大量的能量, 且随着光信号在微环中的传输, 大部 分的能量都消耗在环形波导中，仅有少量的光信号被 耦合到直波导中作为 $\mathrm{B}$ 端口的输出; 而不满足谐振 条件的光信号也从 $\mathrm{B}$ 端口输出 $[9,10]$ 。

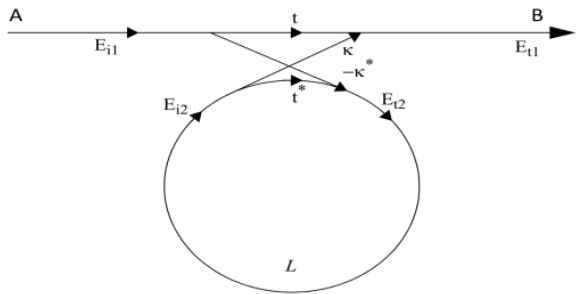

图 1 平行耦合全通型微环谐振腔基本结构耦合机制

以图 1 中最简单的平行耦合全通结构为例，通过 直波导传输的波是入射波与从环耦合到直波导的波 形成的干涉波，入射波 Ei1 的一部分在直波导中传 输, 而其中的一部分场耦合到环形波导中 $[9,10]$ 。类 似地，环形波导中的部分光与直波导中 的光相耦合， 而其余的波围绕环形波导传输。图中 $E_{i 2}$ 为环腔的入 射波, $E_{t 1}$ 和 $E_{t 2}$ 为透射场和耦合场, $t$ 为传输系数, $\kappa$ 为耦合系数, $L$ 为周长, $\alpha$ 为往返损耗, $\beta$ 为 传播常数。

透射场与耦合场关系为:

$\left[\begin{array}{c}E_{t 1} \\ E_{t 2}\end{array}\right]=\left[\begin{array}{cc}t & \kappa \\ -\kappa^{*} & t^{*}\end{array}\right]\left[\begin{array}{l}E_{i 1} \\ E_{i 2}\end{array}\right]$

传输系数和耦合系数关系为

$|t|^{2}=1-|\kappa|^{2}$

当波在环形波导传播时

$E_{i 2}=\alpha E_{t 2} e^{-i \beta L}$ 
结合上述方程可得环内电场与入射场数值比以 及透射场与入射场的数值比:

$$
\begin{aligned}
& \frac{E_{t 2}}{E_{i 1}}=\frac{-i \kappa}{1-t \alpha e^{-i \beta L}} \\
& \frac{E_{t 1}}{E_{i 1}}=\frac{t-\alpha e^{-i \beta L}}{1-t \alpha e^{-i \beta L}}
\end{aligned}
$$

即可得到环内电场和输入端口至透射输出端口 的光强传递函数:

$$
\begin{aligned}
\frac{I_{t 2}}{I_{i 1}} & =\frac{\kappa^{2}}{1+\alpha^{2} t^{2}-2 t \alpha \cos (\beta L)} \\
\frac{I_{t 1}}{I_{i 1}} & =\frac{\alpha^{2}+t^{2}-2 t \alpha \cos (\beta L)}{1+\alpha^{2} t^{2}-2 t \alpha \cos (\beta L)}
\end{aligned}
$$

当环谐振腔处于谐振状态时, 即光能集中在环谐 振腔上, 那么要求 $\beta L=2 \pi m, m$ 为整数, 且 $t=\alpha$ 。

\subsection{App 建立与仿真结果分析}

环型谐振腔耦合建模过程参见 COMSOL Multiphyics 案例库波动光学模块下波导与耦合器中 的《Optical Ring Resonator Notch Filter》, 本文 在这里不做累述。利用 COMSOL Multiphyics 软件可 计算得到在谐振条件下平行耦合全通结构的电场图, 如图 2 所示。

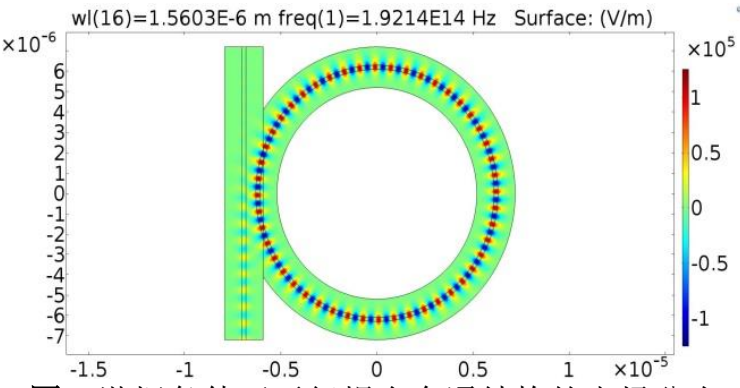

图 2 谐振条件下平行耦合全通结构的电场分布

在谐振状态下, 可发现绝大部分电场从直波导耦 合进入环谐振腔内, 便于学生直观理解耦合过程。但 是如图 3 所示, 利用 COMSOL Multiphyics 软件进行 建模页面, 整个建模计算要相对复杂一些。因此, 利 用 COMSOL Compiler 建立的 App 可极大简化建模过程, 只需要调节相应参数便可完成相关计算及结果分析, 便于学生迅速掌握和理解相关计算过程。App 界面如 图 5 所示。建立好的 APP 可发送至学生电脑, 学生利 用 APP 可完成相关计算以及以利用图形化结论加深 理解, 如图 4 所示。对比图 4 和图 5 可发现 App 中只 需要改变波长、环半径（即改变周长 $\mathrm{L}$ ) 或者折射率 都可以改变谐振状态。例如, 改变环半径 (从 $6.2 \mu \mathrm{m}$ 变为 $9.3 \mu \mathrm{m}$ ), 谐振条件不满足, 直波导内存在大量 电场, 如图 6 所示。通过 APP 可以方便让学生自主进 行参数调整, 通过直观的可视化的模场和透射谱曲线 理解谐振环的工作原理, 加深对整个物理过程本质的 理解。
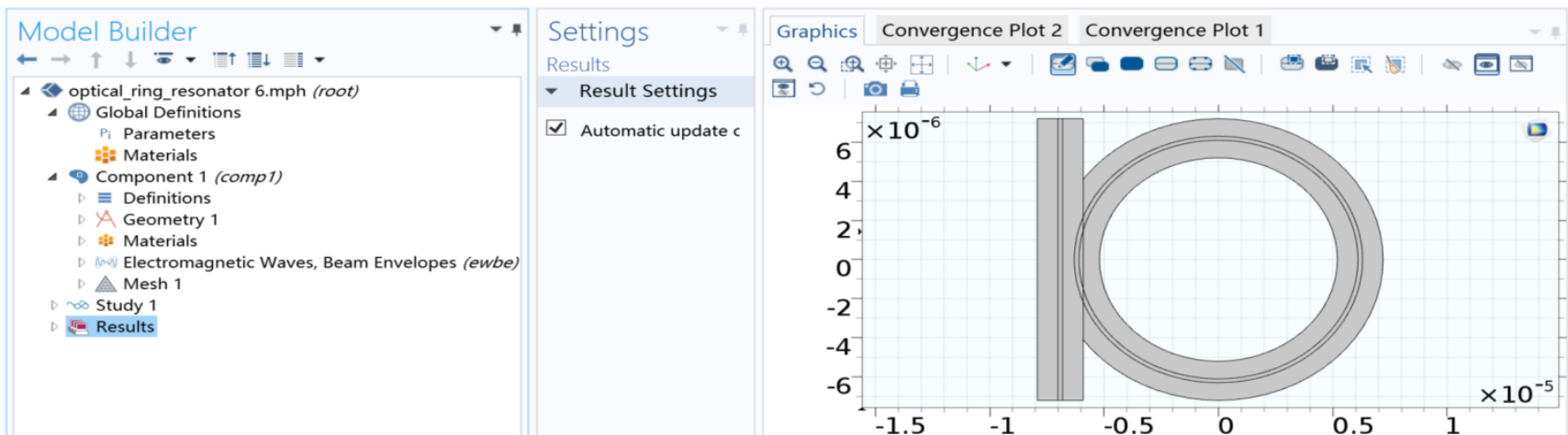

图 3 COMSOL Multiphyics 软件建模页面

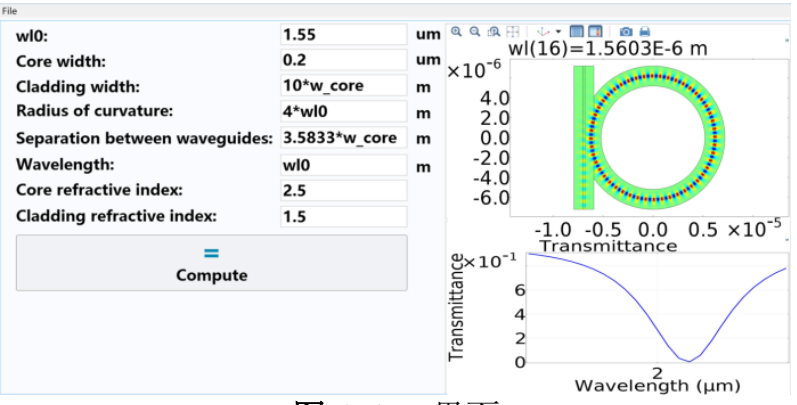

图 4 App 界面

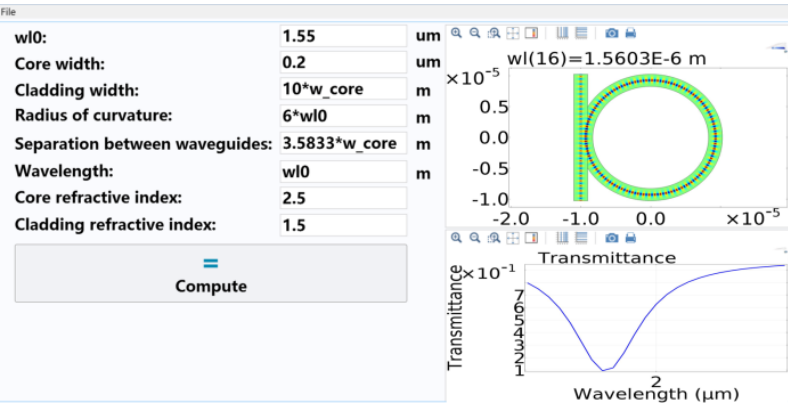

图 5 App 界面上修改环半径 


\subsection{App 教学流程}

教师可将建立好的 App 可发送至学生电脑, 学生可 根据课堂要求进行仿真实验, 也可改变实验参数进行 探索性实验, 在提高学生学习效率与学习兴趣的同时, 进行个性化科研培养。实验结束后, 可将实验结果包 括仿真结果及动画上传。同时也可将相应问题反馈与 老师进行答疑, 建立一个相互沟通的平台, 从而便捷 的解决学生对抽象物理现象的理解。该 App 通过最直 观的方式将多种物理问题通过实物方式呈现, 即能直 观反映很多无法在实验过程观测到的现象, 也能解决 的线下实验器材不足的问题, 还为线上及日常教学带 来了可视化辅助。App 教学流程如图所示。

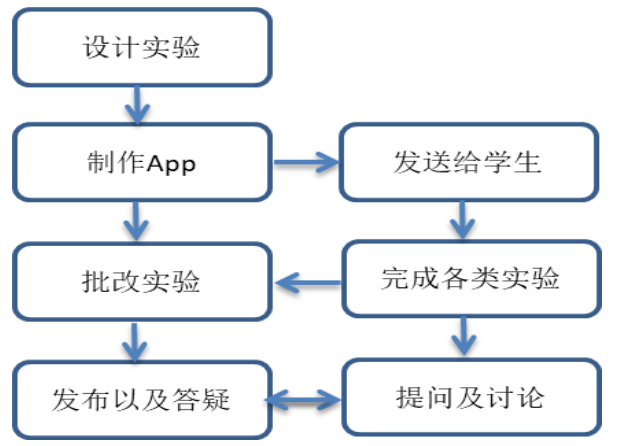

图 7 App 教学流程

\section{3. 结论}

基于 COMSOL Multiphyics App 的在线虚拟仿真 平台可简化复杂的物理建模过程, 使学生通过在简单 明了的界面中进行虚拟实验进一步加深对知识点的 直观理解, 可以很好的提升课堂效率。特别是在当今 疫情条件下的在线课堂以及传统课堂教学要求中, 利 用基于 COMSOL Multiphyics 软件的 App 搭建虚拟仿 真平台不仅能够量身定制对课程知识点的设计讲解, 丰富教学手段, 而且有利于教学资源开放共享, 解决 理论教学和实验课堂的脱节以及多人一组实验仪器 等教学资源不足的问题, 是提高教学质量的新方法新 手段。

\section{项目基金}

本文为兰州大学信息科学与工程学院一流课程 培育计划重点项目资助（2019LZU0049）的阶段性成 果之一。

\section{REFERENCES}

[1] Yao,Y., Zheng,Zh.,Yu.,X. (2020) Current situation and thinking of large-scale online teaching in colleges and universities during Novel Coronavirus Pneumonia Epidemic-Taking Chongqing as an example.J. Chin. J. ICT Edu., (21):5.

[2] Ma, X. (2020) Practice and Thinking of Full Online Remote Hybrid Teaching under Novel Coronavirus Pneumonia Epidemic.J. Univ. Chem.,35(5): 29-32.

[3] Zheng, Y. (2019) Discussion on the Teaching Mode of Electronic Virtual Simulation Experiment under "Internet + Education".J. Popular Sci.\&Techn., 21(10):16-18.

[4] Nie.Y. (2019) Discussions on the Teaching Mode with the Attendance of Virtual Simulations into College Physics Experiments.J. Sci. Edu. Art.C., (12).

[5] Gao. Z. (2020) Current situation and challenge of construction of virtual simulation experimental teaching projects in China.J. Exp. Technol. Mgt., 37(7).

[6] Wan, X. (2017) Research on the application of COMSOL finite element software in the teaching of electromagnetic field theory.J. Heilongjiang Sci., 008(019):79-81.

[7] Liu,H. (2019)Exploration and application of virtual simulation platform and App in fluid mechanics teaching.J. Res.H.E. Eng., (S1): 215-217.

[8] Cheng, L.(1990) Principles and development of optics $[\mathrm{M}]$. Science Press, Beijing.

[9] Rabus, D. G. (2007). Integrated ring resonators. Springer-Verlag Berlin Heidelberg.

[10] Little, B. E., Chu, S. T., Haus, H. A., Foresi, J. A. F. J., \& Laine, J. P. (1997). Microring resonator channel dropping filters. J. Lightwave. Technol., 15(6), 998-1005. 